\title{
Candida Spondylodiscitis: An Unusual Case of Thoracolumbar PAIN WITH ReVIEW Of IMAgING FINDINGS AND DESCRIPTION OF THE Clinical Condition
}

\author{
Francisco M. Torres-Ramos, MD, Kenneth P. Botwin, MD, and Chunilal P. Shah, MD
}

Spondylodiscitis is an unusual but important cause of back pain. Patients with spinal infections typically present with severe sharp aching pain, malaise, fever, and percussion tenderness over the affected area. Early identification of the responsible organism is essential for adequate and prompt treatment.

Fungal spondylodiscitis is extremely rare, and its presentation is insidious in nature. It uncommonly presents with fever or malaise. Clinically, the most reliable physical findings are paravertebral tenderness to palpation and an elevated erythrocyte sedimentation rate. Magnetic resonance imaging is crucial in diagnosing the condition, allowing for early medical intervention.
A case of discitis with adjacent vertebral osteomyelitis (spondylodiscitis) of the thoracic spine due to Candida tropicalis is presented. One of the unusual aspects of this case is that the patient was not immunocompromised.

Kewords: Osteomyelitis, Discitis, back Pain
We present an unusual cause of spondylodiscitis due to candidal infection in a patient with presumed normal immune function. A clinical description, summary of the radiologic findings and review of the literature are provided. Although spondylodiscitis of the thoracolumbar spine is rare, particularly with fungal etiology, prompt diagnosis and appropriate treatment are associated with a good prognosis.

\section{Case Presentation}

A 69-year-old Hispanic female presented with insidious, constant and progressively increasing thoracolumbar pain. The pain was reported for the first time while she was hospitalized for a total shoulder arthroplasty. After surgery, she developed a low-grade fever, which was diagnosed as bronchitis and treated with oral antibiotics for two weeks. The patient was discharged home afebrile but with residual back pain. Two months later, she underwent a cholecystectomy, which was uneventful. Due to her persistent back pain, a CT scan of the lumbar spine was performed which was consistent with severe spinal stenosis at L3-

From Florida Spine Institute, Clearwater, Florida. Address Correspondence: Francisco M Torres-Ramos, MD, 2250 Drew Street, Clearwater, FL 33765. E-mail: contactus@floridaspineinstitute.com Support:There was no external funding in preparation of this manuscript

Conflict of Interest: None
4/L4-5. This was later confirmed with a magnetic resonance imaging (MRI) of the lumbar spine several weeks later.

The patient failed to improve with physical therapy or management by a chiropractor. In the meantime, she continued taking opioid analgesics on a regular basis. During this time, she denied general malaise, fever, or any leg symptoms. Past medical history was non-contributory with no risk factors for human immunodeficiency virus infection or other predisposing factors such as diabetes mellitus, IV drug abuse or chronic alcoholism. There was no history of any prior spinal surgery.

The patient presented to our facility four months after the onset of her symptoms. She complained of pain in the midthoracic and lumbar spine. There was no complaint of radicular pain or neurogenic claudication. On physical examination after the onset of her symptoms, she complained of very tender muscle spasm at the mid thoracic and upper lumbar region. There was loss of spinal mobility. Straight leg raise was negative bilaterally. Neurological examination was normal.

The patient was admitted to the hospital where further workup and imaging studies were done. A diagnostic procedure was performed.

\section{Radiographic Findings}

Thoracic AP and lateral radiographs (Fig. 1) revealed disc space collapse at T8-
9. An MRI of the thoracic spine was obtained on a 1.5 tesla unit. Sagittal and axial T1, T2 and field echo images were obtained. The T1 weighted images (Fig. 2) demonstrated a hypointense/decreased marrow signal replacing the normal marrow of T8 and T9 vertebral bodies with obliteration of the intervening disc. On

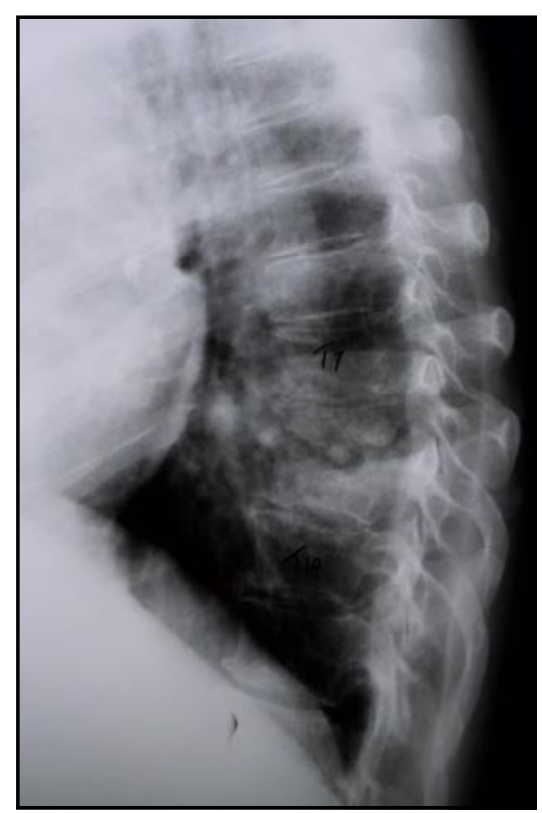

Fig. 1. Lateral radiograph of the thoracic spine revealing disc space collapse at T8/T9 with associated sclerosis in the vertebral bodies of T8 and T9 

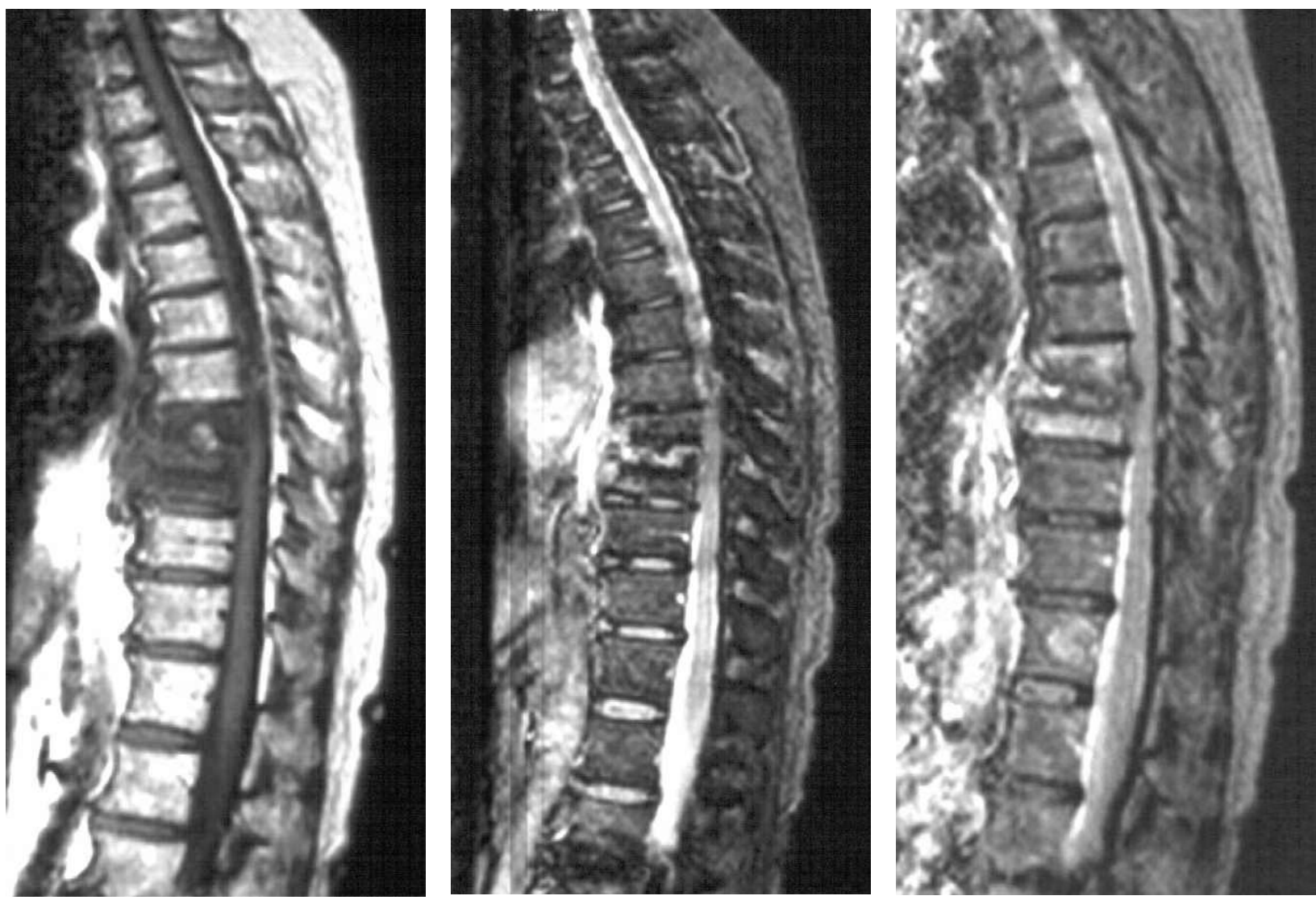

Fig. 2. TI - sagittal MRI image of the thoracic spine reveals decreased or hypointense signal within the T8-T9 disc and associated destruction of vertebral bodies and compromise of the spinal cord at this level. (above left). T2 Sagittal MRI reveals somewhat hyperintense marrow signal. (above right). A field echo image (above center) demonstrates destruction of the disc and sclerosis of the adjoining endplates with pre and paravertebral soft tissue mass.

the T2 weighted images (Fig. 2), the disc and marrow signal changed to somewhat hyperintense signal.

The field echo images (Fig. 2) demonstrated a destroyed disc with increased signal along with destruction and sclerosis of the adjoining endplates. There also was an associated pre- and paravertebral softtissue mass. There was compression of the adjoining ventral aspect of the thoracic cord by a retropulsed bony fragment.

\section{DisCUSSION}

Differential diagnostic possibilities are degenerative disc disease, malignancy and infection. Degenerative disc disease is a complex process, which begins early in life and develops with aging. The pathophysiologic mechanisms are not clearly understood. The process of aging results in the cartilaginous end plate becoming thinner and hyalinized. There is subsequent fissuring and granulation tissue noted in the degenerative disc and end plate as well. There is a change in the con- tent of the type II collagen, which increases in the annulus. There is also an increase in the ratio of keratin sulphate to chondroitin sulphate (1).

The appearance of the degenerative disc and degenerative end plate changes observed on MRI imaging has been well described by Modic et al (2). They described a classification scheme for disc disease by dividing it into type I, type II and type III changes.

- $\quad$ Type I includes end plate changes that represent vascularized marrow and are seen as low intensity on $\mathrm{T} 1$ weighted images and high intensity T2 weighted images.

- Type II changes represent more chronic discogenic disc disease with a presence of fatty marrow and are characterized by high signal intensity on $\mathrm{T} 1$ weighted images and are isointense or slightly hyperintense on T2 images.

- Type III changes represent dense bone devoid of marrow signal resulting in hypointensity on $\mathrm{T} 1$ and T2 weighted images.

With the MRI findings described in this case, an extradural tumor would have to be considered in the differential diagnosis. Usually, with unenhanced MRI scans there is good delineation of an extradural lesion. The vertebral body lesions tend to be of low intensity on $\mathrm{T} 1$ weight images whereas, with a T2 image there would be high signal intensity (3). Gadolinium contrast may help to delineate a neoplastic processes. In malignant lesions, which are most commonly metastatic there is usually involvement of the vertebral body and pedicle. The disc space is spared and end plates are usually intact. There may be associated destruction of the bony margin with an intraspinal and/ or paraspinal soft tissue mass usually at the vertebral body level, whereas inflammatory phlegmon or abscess with discitis is usually centered at the disc space lev- 
el. The extradural mass effect seen with degenerative disc disease is secondary to osteophytes and associated disc bulge or herniated nucleus pulposus.

The third possible diagnosis is an infectious process (e.g., discitis, osteomyelitis, spondylodiscitis). Normally, radiographic changes in vertebral osteomyelitis are present two to eight weeks after the onset of symptoms (4). MRI is more sensitive, specific and accurate than radioisotope bone scan or CT scan for early recognition and localization of infectious disease. Lifeso et al (5) found MRI to be $96 \%$ sensitive, $92 \%$ specific and $94 \%$ accurate in the diagnosis of vertebral osteomyelitis. In addition, MRI is an excellent way of demonstrating the presence of epidural or paraspinal extension of the infection. Thus, MRI studies may lead to early diagnosis, which in combination with adequate and prompt medical treatment may reduce the need for surgical intervention.

Based on the MRI findings in our patient, described in Fig. 2, an infectious process was considered likely. Note that the previous MRI was limited to the lumbar spine and showed lumbar spinal stenosis. In addition, blood tests revealed an elevated erythrocyte sedimentation rate (ESR) of 66 and an elevated alkaline phosphatase. Serum protein electrophoresis (SPEP) showed Alpha II elevation consistent with an inflammatory process.

The patient was admitted to the hospital where she underwent vertebrectomy, debridement and fusion. Cultures from the spine were reported as positive for fungi, which eventually grew Candida tropicalis. She was placed on Amphotericin B and Vancomycin. Further laboratory studies failed to show human immunodeficiency virus antibody with no defect in immune function. The patient was discharged home in a brace. After twelve weeks of antibiotic therapy she did well. One year later, she was healthy with no recurrence of her infection.

Spondylodiscitis of the thoracolumbar spine is rare. The diagnosis tends to be overlooked, which can lead to serious sequelae. If identified early it can be appropriately treated with a good prognosis. On physical examination there can be decreased range of motion, muscle spasm or even spinal deformity. Patients with spinal infections usually present with a sharp aching pain, general malaise, fever, and percussion tenderness over the affected area. If severe and untreated, a neurologic deficit may be present.

The infections are most commonly bacterial (gram positive or gram negative) Anaerobic, Mycobacteria and rarely fungal (6). Fungal spondylodiscitis is a rare condition. However, its incidence appears to be on the rise, probably related to the increase number of immunocompromised patients or intravenous drug abusers (7). This condition usually occurs through hematogenous spread from infected foci in the body or by direct extension from instrumentation or surgery (8).

Candida osteomyelitis overall is a rare entity. A review of the literature done in 1987 revealed only 32 case reports of Candida osteomyelitis in adults (9), and 59 by Miller and Mejicano in 2001 (10). Of these, only 11 cases were from Candida tropicalis and 9 cases occurred in immunocompromised patients.

Of the 59 cases described by Miller and Mejicano (10), 33 cases involved only the lumbar spine, 17 involved the thoracic spine, 3 the cervical spine, and 6 both the thoracic and lumbar spine. There were eleven cases of Candida tropicalis spondylodiscitis, 7 involving the thoracic spine, 3 the lumbar spine and one both the cervical and thoracic spine (10).

When Candida involves the spine, it is usually centered around the intervertebral disc space with narrowing of the disc cartilage, causing destruction and lysis of the vertebral endplates and underlying vertebral bone (9).

This condition is usually insidious in nature. It is uncommon to have associated symptoms such as low-grade fever or chills. The most useful clinical finding identifying spondylodiscitis is point tenderness over the affected area with associated paravertebral tenderness (12). The sedimentation rate is usually elevated and occasionally the alkaline phosphatase is increased.

Predisposing factors associated with this condition include: diabetes mellitus, extremes of age, IV drug abuse, intravenous access, discography, infective endocarditis, urinary tract infections chronic alcoholism and immunocompromised states.

In the English literature there have been two previous reports of Candida tropicalis osteomyelitis in an immunocompetent adult $(10,12,13)$. One patient had candidemia after a staghorn calculus, which required a nephrectomy (14). The case described by Kashi Motio et al (15) was in a patient who had a blood transfusion for melena 44 months prior to being admitted for thoracic pain. In both cases surgical treatment along with antifungal therapy relieved the condition $(14,15)$. The prognosis appears to be favorable, with an overall cure rate of candidal vertebral osteomyelitis of $85 \%$ (10).

\section{Conclusion}

Fungal spondylodiscitis is rare, particularly in immunocompetent patients. Physical findings may be minimal, although spinal tenderness is usually evident and the ESR is frequently elevated. MRI imaging is crucial in the diagnosis of spondylodiscitis, provided that the symptomatic part of the spine is examined. This can aid in early diagnosis, surgical debridement and antibiotic treatment. Amphotericin B remains the primary antifungal agent. The outcome in our case was good, with complete recovery after surgical debridement and 12 weeks of antibiotics. This is in agreement with the literature, which reports a favorable outcome in the majority of cases.

Author Affiliation:
Francisco M. Torres-Ramos, MD
Florida Spine Institute
2250 Drew Street
Clearwater, FL 33765
E-mail: contactus@floridaspineinsti
tute.com
Kenneth P. Botwin, MD
Florida Spine Institute
2250 Drew Street
Clearwater, FL 33765
E-mail: contactus@floridaspineinsti
tute.com
Chunilal P. Shah, MD
Florida Spine Institute
2250 Drew Street
Clearwater, FL 33765
E-mail: contactus@floridaspineinsti
tute.com

\section{References}

1. Adams P, Eyve DR, Muid H. Biochemical aspects of development and ageing of human lumbar intervertebral discs. Rheumatol Rehabil 1977; 16:22-29.

2. Modic MT, Steinberg PM, Ross JS. Degenerative disc disease: Assessment of changes in vertebral body marrow with MR imaging. Radiology 1988; 166:193-199.

3. Carmody RF, Yang DJ, Seeley GW et al. Spi- 
nal cord compression due to metastatic disease: Diagnosis with MR imaging versus myelography. Radiology 1989; 173: 225-229.

4. Kramer J, Stiglbauer RN, Wimberger D et al. MRI of spondylitis. Bildgebung 1992; 59:147-151.

5. Lifeso RM. Pyogenic spinal sepsis in adults. Spine 1990; 15:1265-1271.

6 Schwartz ST, Spiegel M, Hog JR. Bacterial vertebral osteomyelitis and epidural abscess. Semin Spine Surg 1990; 2:98.

7. Fang D, Cheung KC, Dos Remedios DN et al. Pyogenic vertebral osteomyelitis: Treatment of anterior spinal debridement and fusion. J Spinal Disord 1994; 17:173-180.

8. Smith AS, Blaser SI. Infections and inflammatory processes of the spine. Radiol Clin North Am 1991; 29:809-827.

9. Gathe JC, Harris RL, Garland B et al. Candida osteomyelitis: Report of five cases and review of the literature. Am J Med 1987; 82:927-937.

10. Miller DJ. Vertebral osteomyelitis due to Candida species: Case report and literature review. Clin Infect Dis 2001; 33:523-530.

11. McCain GA, Harth M, Bell DA et al. Septic discitis. J Rheumatol 1981; 8:100-109.

12. Smith A, Blaser S. Infections and inflammatory processes of the spine. Radiol Clin
North Am 1991; 29:809-827.

13. Friedman BC, Simon GL. Candida Vertebral Osteomyelitis: Report of three cases and a review of the literature. Diagn Microbiol Infect Dis 1987; 8:31-36.

14. Sugar AM, Saunders C, Diamond RD. Successful treatment of candida osteomyelitis with fluconazole. A non-comparative study of two patients. Diagn Microbiol Infect Dis 1990; 13:517-520.

15. Kashimoto T, Kitagawa H, Kachi H. Candida tropicalis vertebral osteomyelitis and discitis. A case report and discussion on the diagnosis and treatment. Spine 1984 12:57-61. 